\title{
DYNAMIC SIMULATION OF A NOVEL "BROOMSTICK" HUMAN FORWARD FALL MODEL AND FINITE ELEMENT ANALYSIS OF THE RADIUS UNDER THE IMPACT FORCE DURING FALL
}

\author{
Dariusz Grzelczyk, Pawee Biesiacki, Jerzy Mrozowski, Jan Awrejcewicz \\ Lodz University of Technology, Department of Automation, Biomechanics and Mechatronics, Eódź, Poland \\ e-mail: dariusz.grzelczyk@p.lodz.pl; pawel.biesiacki@dokt.p.lodz.pl; jerzy.mrozowski@p.lodz.pl; jan.awrejcewicz@p.lodz.pl
}

\begin{abstract}
The paper presents dynamic simulation and experimental identification of a human forward fall model describing the process of "falling like a broomstick" on the outstretched arms. The model implemented in Mathematica allows one to estimate time histories of the ground reaction force in different scenarios of the fall process. These time series are applied as time-varying load conditions to the numerical analysis of the human radial bone model created from the computed tomography data. Finally, the obtained numerical results indicate that the strain criterion seems to be more useful for estimating the radius fracture site in comparison to the stress criterion.
\end{abstract}

Keywords: forward fall, ground reaction force, bone strength, fracture, distal radius

\section{Introduction}

Falls are common accidents in human daily life. They are severe and inevitable threats during the walking process of the bipedal movement. Despite all population groups are exposed to these risk factors, falls are the most serious social health problem among the elderly (Heijnen and Rietdyk, 2016). As a result, the risk of injuries to hands, torso, head/neck and/or other parts of the human body is possible. These injuries can eventually lead to death, chronic pain, disability and/or loss of independence (Robinovitch et al., 2013).

The vast majority of cases of upper extremity injuries occur as a result of a fall with a direct impact on the extended arms which are exposed to dynamical impact forces (Nevitt and Cummings, 1993; Palvanen et al., 2000). Distal radius fractures are common in eldery women with osteoporosis due to their compromised bone density/quality as well as probably due to the increased risk of falling in this population group. Fractures of the forearm bones represent nearly $20 \%$ of all reported fractures worldwide, and the most common type of fracture is the so-called Colles' fracture as an injury of distal radius of the forearm (Johnell and Kannis, 2006).

The aforementioned upper extremity injuries may be a result of forward falls, backward falls or side falls. Colles' fracture, as an injury of the radius, is a direct result of exceeding the maximum value of the force allowable for this bone. For instance, distal radius fractures at a mean force equal to $2260 \pm 1010 \mathrm{~N}$ were observed by Frykman using 48 cadaveric bones of average age of 65 years (Frykman, 1967). Spadaro and his co-investigators obtained the mean strength of a bone fracture at the level of $1640 \pm 980 \mathrm{~N}$ (Spadaro et al., 1994). Kim and Ashton-Miller (2009) adopted the value equal to $2400 \mathrm{~N}$ as a distal radius fracture threshold. Also in one of the recent papers by Burkhart et al. (2013), the estimated values of force causing fracture of the studied bones derived from cadavers were approximately equal to $2150 \mathrm{~N}$. 


\section{Literature review of the forward fall models}

A forward fall is known as the most frequent type of fall, and more than a half of all falls among the elderly occur in the forward direction (Nevitt and Cummings, 1993; O'Neill et al., 1994; Vellas et al., 1998). Recent decades have brought different models related to the impact of the upper extremities to the ground as a result of a forward or backward fall. One of the simplest effective mechanical models of the human falling motion is a single-degree-of-freedom (DoF) linear mass-spring-damper mechanical system subjected to a sudden velocity input or an impulse force input. Such a model can be readily extended to systems with many DoFs. Below we are focused only on the three most well-known, widely available and cited forward fall models.

A fall model proposed by Chiu and Robinovitch (1998) applies to the human forward fall from a low height on the outstretched and fully extended upper extremities as the worst-case scenario of such a fall. It is constructed based on a 2-DoFs lumped-parameter mechanical system containing elastic and damping elements responsible for the operation of human muscles. Davidson et al. (2006) completed a study with the aim of developing a "risk factor" value in order to determine whether someone will sustain the radius fracture based on the characteristics of their fall. The mentioned study used a two mass-spring-damper model, referring to the experimental data obtained from 45 clinical cases of children falling off of playground equipment.

DeGoede and Ashton-Miller (2003) employed Adams software to devolop a half-body symmetric model of the human forward fall consisting of five segments (legs, torso with head and neck, upper arm, forearm and hand). In that model, the movement of the hand and the pivot point in the ankle toe are fixed, and friction is not taken into account. Moreover, the wrist, elbow and hip joints are adopted as flat joints without friction, whereas the shoulder joint connecting the arm to the body via massless blades is modelled as a linear frictionless sliding joint with the movement axis perpendicular to the longitudinal body axis. The main goal of the authors was to study the possibility of injury in older women. Therefore, to determine the model parameters, baseline height and weight equal to $1.63 \mathrm{~m}$ and $62 \mathrm{~kg}$, respectively, were used (Kroemer et al., 1997).

Kim and Ashton-Miller (2009) proposed another flat model of the forward fall as a two DoFs system constructed based on a mechanical model of a double pendulum rotating freely around a pivot corresponding to the ankles of the lower human extremities. To provide a mathematical model, the mechanical system was reduced to a system of linear translational movements with 2-DoFs with spring-damper elements responsible for attenuation action of the human muscles. Numerical simulation for the fall height of $1.5 \mathrm{~m}$ showed that the maximum impact force was doubled from $1250 \mathrm{~N}$ to a value of $2610 \mathrm{~N}$, depending on the fall scenario. In this way, the authors showed that for the same fall conditions (the same faller falling from the same height), too rapid movement of the arms in comparison to the rest of the body could cause the occurence of an excessive force acting on the upper extremity more than $2350 \mathrm{~N}$, which caused a fracture of the distal radius.

To conclude, it can be stated that, in general, the considered falling process was usually mathematically modelled based on flat and linear mechanical systems consisting of two rigid bodies with masses moved by transverse motion connected by linear spring-damper elements. These models were usually presented as a set of the second-order ordinary differential equations of motion (Chiu and Robinovitch, 1998), but also the appropriate equations were written in the state space (Kim and Ashton-Miller, 2009). On the other hand, in the case of a more complex mechanical model, numerical simulations were performed only using commercial Adams software (DeGoede and Ashton-Miller, 2003). In the present paper, we propose our own mathematical model of the human forward fall on the outstretched arms. In addition, unlike previous models met in the literature, our model takes into account a direct influence of different human speed 
just before the trip over an obstacle and starting the falling process, which affects the final force of impact of the upper extremity on the ground.

\section{The proposed "broomstick" forward fall model}

\subsection{Mathematical modelling}

The human "like a broomstick" forward fall on the outstretched arms is schematically shown in Fig. 1a. Equations of motion of the analysed system have been obtained by the Newton-Euler method, and Free Body Diagrams of the system are shown in Fig. 1b.

(a)

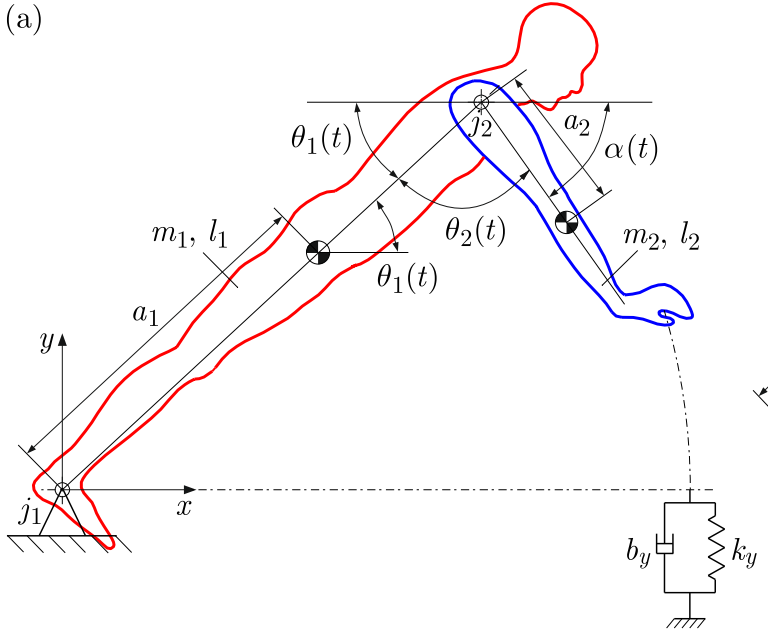

(b)

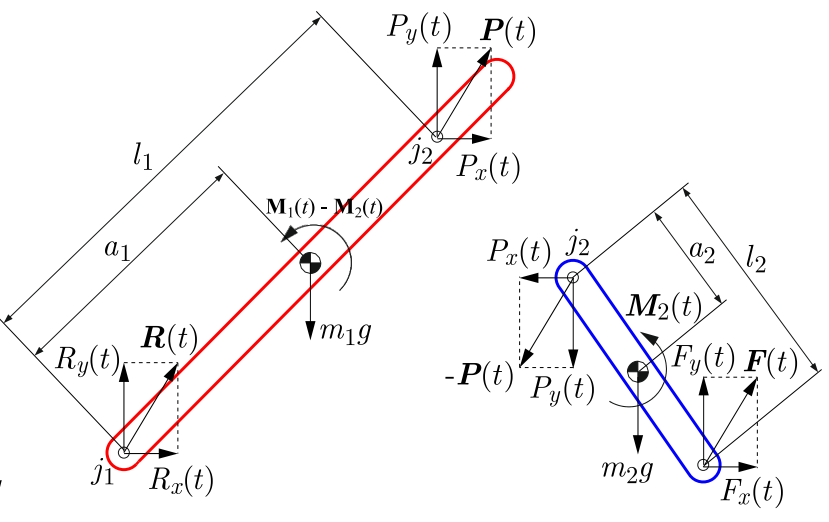

Fig. 1. Mechanical modelling of the forward fall process: (a) the proposed forward fall biomechanical model as a planar mechanical system with 2-DoFs embedded in the Cartesian coordinate system;

(b) Free Body Diagrams of the system

The model consists of two rigid bodies, 1 and 2, with masses $m_{1}, m_{2}$ and moments of inertia $I_{1}, I_{2}$ around centres of gravity of the bodies, respectively. Body 1 corresponds to the human torso with legs, neck and head, whereas body 2 corresponds to the human upper extremities (including arms, forearms and hands). The parameters $a_{1}$ and $a_{2}$ represent the distances between the gravity centres of individual bodies and their rotation axes, whereas $l_{1}$ and $l_{2}$ denote the total lengths of the mentioned bodies, respectively. The angle $\theta_{1}(t)$ denotes the angle between the horizontal $x$ axis and the longitudinal axis of body 1 . The angle $\theta_{2}(t)$ is the angle measured from the axis of body 1 to the axis of body 2 . The ground with non-linear contact law is characterised by stiffness and damping coefficients $k_{y}$ and $b_{y}$, respectively.

In this model, we take the vectors $\boldsymbol{\theta}_{1}(t)=\left[0,0, \theta_{1}(t)\right]^{\mathrm{T}}, \boldsymbol{\theta}_{2}(t)=\left[0,0, \theta_{2}(t)\right]^{\mathrm{T}}$ of the angles in the joints $\mathrm{j} 1$ and $\mathrm{j} 2$, vectors $\mathbf{r}_{C 1}(t), \mathbf{r}_{C 2}(t)$ of displacements of gravity centres of the first and the second body, as well as the vectors $\mathbf{l}_{1}(t), \mathbf{l}_{2}(t)$ of displacements of the joint $\mathrm{j} 2$ and the end of body 2 which can impact to the ground, respectively, in the following form

$$
\begin{aligned}
& \mathbf{r}_{C 1}(t)=\left[x_{1}(t), y_{1}(t), 0\right]^{\mathrm{T}}=\left[a_{1} \cos \theta_{1}(t), a_{1} \sin \theta_{1}(t), 0\right]^{\mathrm{T}} \\
& \mathbf{r}_{C 2}(t)=\left[x_{2}(t), y_{2}(t), 0\right]^{\mathrm{T}}=\left[l_{1} \cos \theta_{1}(t)+a_{2} \cos \alpha(t), l_{1} \sin \theta_{1}(t)-a_{2} \sin \alpha(t), 0\right]^{\mathrm{T}} \\
& \mathbf{l}_{1}(t)=\left[l_{1} \cos \theta_{1}(t), l_{1} \sin \theta_{1}(t), 0\right]^{\mathrm{T}} \\
& \mathbf{l}_{2}(t)=\left[l_{1} \cos \theta_{1}(t)+l_{2} \cos \alpha(t), l_{1} \sin \theta_{1}(t)-l_{2} \sin \alpha(t), 0\right]^{\mathrm{T}}
\end{aligned}
$$

where $\alpha(t)=180^{\circ}-\theta_{1}(t)-\theta_{2}(t)$. The forces $\mathbf{Q}_{1}=\left[0,-m_{1} g, 0\right]^{\mathrm{T}}$ and $\mathbf{Q}_{2}=\left[0,-m_{2} g, 0\right]^{\mathrm{T}}$ are the gravity forces acting on the gravity centres of bodies 1 and 2 , respectively, with the acceleration 
of gravity $g\left(g=9.81 \mathrm{~m} / \mathrm{s}^{2}\right)$. The force $\mathbf{R}(t)=\left[R_{x}(t), R_{y}(t), 0\right]^{\mathrm{T}}$ is the reaction force in the joint $\mathrm{j} 1$. The unknown force in the joint $\mathrm{j} 2$ and presented in the Free Body Diagrams (see Fig. 1b) is denoted as $\mathbf{P}(t)=\left[P_{x}(t), P_{y}(t), 0\right]^{\mathrm{T}}$. Finally, the force $\mathbf{F}(t)=\left[F_{x}(t), F_{y}(t), 0\right]^{\mathrm{T}}$ is the ground reaction force acting on body 2 at the moment of its impact to the ground.

Let $\mathbf{M}_{1}(t)=[0,0,0]^{\mathrm{T}}$ and $\mathbf{M}_{2}(t)=\left[0,0, M_{2}(t)\right]^{\mathrm{T}}$ denote the torques generated in the joints $\mathrm{j} 1$ and $\mathrm{j} 2$, respectively. As a result, for the two considered free bodies presented in Fig. 1b, we can write the following equations of motion in the vector form

$$
\begin{aligned}
& m_{1} \ddot{\mathbf{r}}_{C 1}(t)=\mathbf{R}(t)+\mathbf{Q}_{1}+\mathbf{P}(t) \\
& I_{1} \ddot{\boldsymbol{\theta}}_{1}(t)=\mathbf{M}_{1}(t)-\mathbf{M}_{2}(t)-\mathbf{r}_{C 1}(t) \times \mathbf{R}(t)+\left[\mathbf{l}_{1}(t)-\mathbf{r}_{C 1}(t)\right] \times \mathbf{P}(t) \\
& m_{2} \ddot{\mathbf{r}}_{C 2}(t)=-\mathbf{P}(t)+\mathbf{Q}_{2}+\mathbf{F}(t) \\
& I_{2} \ddot{\boldsymbol{\theta}}_{2}(t)=\mathbf{M}_{2}(t)-\left[\mathbf{l}_{1}(t)-\mathbf{r}_{C 2}(t)\right] \times \mathbf{P}(t)+\left[\mathbf{l}_{2}(t)-\mathbf{r}_{C 2}(t)\right] \times \mathbf{F}(t)
\end{aligned}
$$

Equations (3.2) can be reduced to the scalar form

$$
\begin{gathered}
I_{1} \ddot{\theta}_{1}(t)=-M_{2}(t)+a_{1} R_{x}(t) \sin \theta_{1}(t)-a_{1} R_{y}(t) \cos \theta_{1}(t) \\
\quad+\left(l_{1}-a_{1}\right) P_{y}(t) \cos \theta_{1}(t)-\left(l_{1}-a_{1}\right) P_{x}(t) \sin \theta_{1}(t) \\
I_{2} \ddot{\theta}_{2}(t)=M_{2}(t)+a_{2} P_{y}(t) \cos \alpha(t)+a_{2} P_{x}(t) \sin \alpha(t) \\
\quad+\left(l_{2}-a_{2}\right) F_{y}(t) \cos \alpha(t)+\left(l_{2}-a_{2}\right) F_{x}(t) \sin \alpha(t)
\end{gathered}
$$

where

$$
\begin{aligned}
& P_{x}(t)=F_{x}(t)-m_{2} \ddot{x}_{2}(t) \\
& P_{y}(t)=F_{y}(t)-m_{2} \ddot{y}_{2}(t)-m_{2} g \\
& R_{x}(t)=m_{1} \ddot{x}_{1}(t)-P_{x}(t)=m_{1} \ddot{x}_{1}(t)+m_{2} \ddot{x}_{2}(t)-F_{x}(t) \\
& R_{y}(t)=m_{1} \ddot{y}_{1}(t)+m_{1} g-P_{y}(t)=m_{1} \ddot{y}_{1}(t)+m_{2} \ddot{y}_{2}(t)+m_{1} g+m_{2} g-F_{y}(t)
\end{aligned}
$$

At the time of tripping over an obstacle, a human instinctively and quickly pulls his arms to the front in order to arrest and/or to absorb the fall. This process is described by the time histories of the angle $\theta_{2}(t)$, which can be estimated based on the kinematics of the falling process registered with the use of a digital camera. Based on the second equation of (3.3) and the function $\theta_{2}(t)$ obtained in this way, we can calculate the torque $M_{2}(t)$ generated by arms in the joint shoulder during the falling process. Taking the torque $M_{2}(t)$ in the first Eq. of (3.3), one obtains eventually the following equation of motion around the joint $\mathrm{j} 1$

$$
\begin{aligned}
& I_{1} \ddot{\theta}_{1}(t)+I_{2} \ddot{\theta}_{2}(t)=a_{1} R_{x}(t) \sin \theta_{1}(t)-a_{1} R_{y}(t) \cos \theta_{1}(t)+\left(l_{1}-a_{1}\right) P_{y}(t) \cos \theta_{1}(t) \\
& \quad-\left(l_{1}-a_{1}\right) P_{x}(t) \sin \theta_{1}(t)+a_{2} P_{y}(t) \cos \alpha(t)+a_{2} P_{x}(t) \sin \alpha(t) \\
& \quad+\left(l_{2}-a_{2}\right) F_{y}(t) \cos \alpha(t)+\left(l_{2}-a_{2}\right) F_{x}(t) \sin \alpha(t)
\end{aligned}
$$

with the function $\theta_{1}(t)$ as a solution to this equation of motion.

\subsection{Ground reaction force}

To predict the vertical ground reaction force $(\mathrm{GRF}) F_{y}(t)$, a non-linear model of impact at the wrist-ground interface has been employed in the form

$$
F_{y}(t)=k_{y}|y(t)|^{3}\left(1-b_{y} \dot{y}(t)\right) J(-y(t))
$$

where parameters $k_{y}, b_{y}$ denote the ground stiffness and damping coefficients in the vertical direction, respectively, $y(t)=l_{1} \sin \theta_{1}(t)-l_{2} \sin \alpha(t)$, and the function $J(-y(t))$ is the step function defined as

$$
J(-y(t))=\left\{\begin{array}{lll}
1 & \text { if } \quad y(t)<0 \\
0 & \text { if } \quad y(t) \geqslant 0
\end{array}\right.
$$


For instance, this formulation of GRF had been used previously to model the heel strike in running (Gerritsen et al., 1995) and to model GRF at the hand-ground interface (DeGoede and Ashton-Miller, 2003). In our model, we have not included the horizontal force component $F_{x}(t)$ which is required to prevent the hand from sliding. However, the total GRF is less than 5\% greater than the vertical GRF (DeGoede and Ashton-Miller, 2003).

\subsection{Initial conditions}

The considered model has been reduced to a single DoF model with the function $\theta_{1}(t)$ as the solution and the following initial conditions: $\theta_{1}(0)$-initial angular position, and $\dot{\theta}_{1}(0)$-initial angular velocity. At the beginning of the trip $(t=0)$, a human is usually in the standing position, and thus we take $\theta_{1}(0)=90^{\circ}$ (see Fig. 1a). The initial angular velocity $\dot{\theta}_{1}(0)$ is estimated based on the transverse human speed during walking just before the moment of the trip. For this reason, we assume that the linear speed of the human gait decreases from $v_{0}$ to 0 , and the rotational velocity $\dot{\theta}_{1}(t)$ increases from 0 to $\dot{\theta}_{1}(0)$ at the same time. Taking into account the principle of conservation of momentum, rotary motion of the human body around the pivot axis is governed as

$$
I \frac{\Delta \dot{\theta}_{1}(t)}{\Delta t}=r F \Rightarrow \Delta \dot{\theta}_{1}(t)=-\frac{\left(m_{1}+m_{2}\right) v_{0} r}{I}=\dot{\theta}_{1}(0)
$$

where $F \Delta t$ is a force impulse causing rotation of the human body around the rotation axis placed in feet (ankles), $I$ is the moment of inertia of the human body around the pivot axis and $r$ is the distance between the human gravity centre and the pivot axis with the upper extremities adjusted along the body (typical position of the human body during walking). On the contrary to the previous investigations met in the literature, the assumption adopted in this paper allows one also to study kinematic and dynamic parameters during the falling process for different walking speeds of the faller just before the moment of the trip over an obstacle.

\section{Identification of the fall model parameters}

\subsection{GrabCAD model of the human body}

To determine the human body parameters required in the proposed fall model, the full 3D scanned human body model based on GrabCAD (2016) (see Fig. 2) as well as the appropriate AutoDesk Inventor commands have been used. The mass of the whole body is calculated assuming that the average density equals to $\rho=1050 \mathrm{~kg} / \mathrm{m}^{3}$, and the model parameters are: $a_{1}=1.01 \mathrm{~m}, a_{2}=0.22 \mathrm{~m}, l_{1}=1.4 \mathrm{~m}, l_{2}=0.53 \mathrm{~m}, r=1.03 \mathrm{~m}, m_{1}=61.0 \mathrm{~kg}, m_{2}=7.4 \mathrm{~kg}$, $I_{1}=9.9 \mathrm{~kg} \cdot \mathrm{m}^{2}, I_{2}=0.232 \mathrm{~kg} \cdot \mathrm{m}^{2}, I=82.86 \mathrm{~kg} \cdot \mathrm{m}^{2}$.

\subsection{Kinematics of the fall process}

The kinematic analysis of the faller between the moment of tripping over an obstacle and hitting his hands to the ground were observed using an Optitrack optoelectronic motion analysis system installed at the Department of Automation, Biomechanics and Mechatronics, Lodz University of Technology, Lodz, Poland. The front panel of the computer program used to operate this system with the location of the individual markers on the faller's body is presented in Fig. 3, where the process of forward fall from the standing position to the ground is shown. Before the experimenal test, the volunteer (one of the authors of this paper) has been instructed to safely fall "like a broomstick". The fall of the human body was performed using a certificated soft insurance mattress with the full size $2 \mathrm{~m} \times 2 \mathrm{~m} \times 0.3 \mathrm{~m}$, and thus there was no a direct threat of injury to the upper extremities and other body parts. 


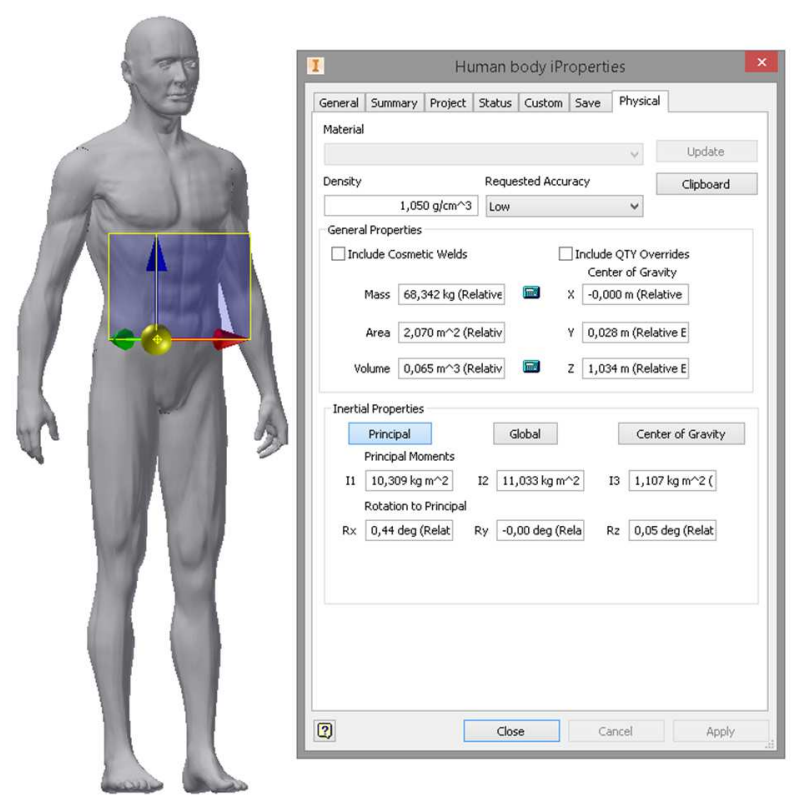

Fig. 2. Three-dimensional scanned human body model used for the estimation of its geometrical and mechanical properties (GrabCAD, 2016)

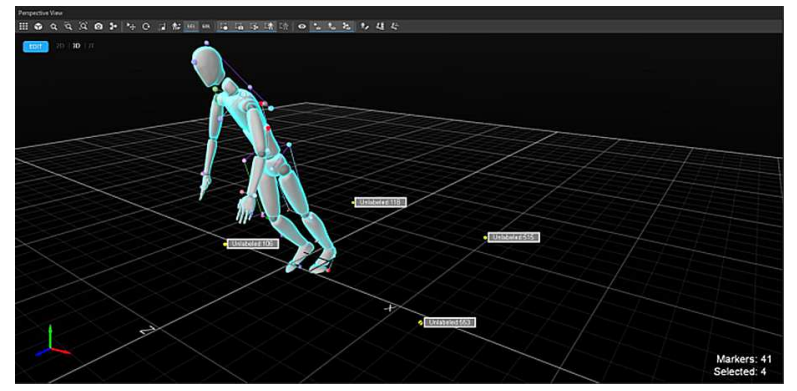

Fig. 3. Forward fall from the standing position to the soft mattress observed by Optitrack system using 37 passive reflective markers distributed on the faller's body

Using Optitrack system we observed that the faller extends his arms from initial position $\theta_{20} \approx 5^{\circ}$ to the final position $\theta_{2 \max } \approx 80^{\circ}$ at the moment of the impact to the ground. That is why, in all further presented investigations we use the time history of angle $\theta_{2}(t)$ described by the formula

$$
\theta_{2}(t)= \begin{cases}\theta_{20}+\left(\theta_{2 \max }-\theta_{20}\right) \sin ^{2}(\lambda t) & \text { if } t \leqslant T \\ \theta_{2 \max } & \text { if } t>T\end{cases}
$$

where $T$ denotes duration of the fall (the time between tripping and hitting the ground), whereas $\lambda$ corresponds to the speed of movements of the faller's arms. The parameters $\lambda$ and $T$ strongly depend on the walking speed of the faller before the trip. The averaged time history of the angle $\theta_{2}(t)$ obtained from the experiment for $v_{0}=1.5 \mathrm{~m} / \mathrm{s}$ and its approximation are presented in Fig. 4.

\subsection{Hand-ground contact parameters}

Figure 5a shows the schematics (the first frame of our animation created in Mathematica) of the initial configuration of the subject falling in the forward direction from the initial shoulder height of $1 \mathrm{~m}$ (DeGoede and Ashton-Miller, 2003). The red line represents the torso with legs, the blue line represents upper extremities, whereas the green circle is the head of the faller. 


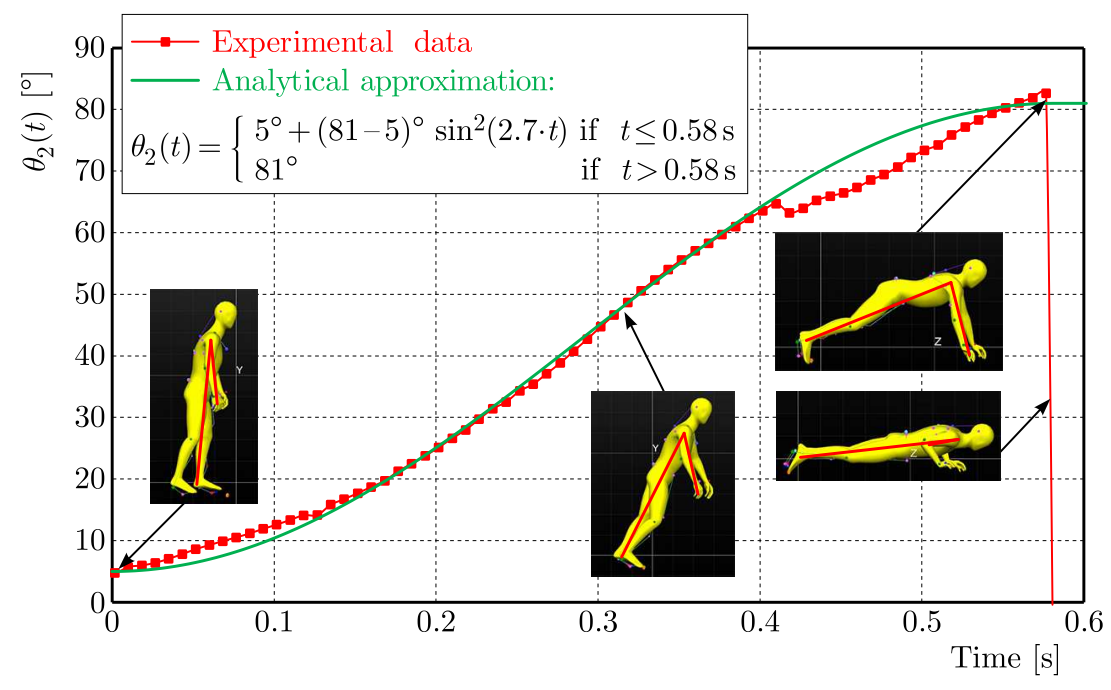

Fig. 4. Time history of the angle $\theta_{2}(t)$ (red points and red curve) obtained from the experiment and its approximation by an analytical smooth function (green curve). In this experiment, the faller started to fall from not a fully standing position $\left(\theta_{1}(0)<90^{\circ}\right)$

The presented initial position was obtained for the following initial conditions: $\theta_{1}(0)=45^{\circ}$, $\dot{\theta}_{1}(0)=0$ and $\theta_{2}(t)=83.5^{\circ}=$ const. During numerical experiments we tested different values of the parameters $k_{y}$ and $b_{y}$ to obtain GRF which corresponds (both from a qualitative and quantitative point of view) to the GRFs presented by DeGoede and Ashton-Miller (2003). In that paper, the authors tested five healthy young male volunteers aged between 22 and 28 years with the average body mass of $72 \pm 7 \mathrm{~kg}$ and the overall height of $173 \pm 3 \mathrm{~cm}$ (DeGoede and Ashton-Miller, 2002). Finally, the best degree of fit was obtained for $k_{y}=50000 \mathrm{~N} / \mathrm{m}^{3}$ and $b_{y}=0.6 \mathrm{~s} / \mathrm{m}$ (the obtained results are shown in Fig. $5 \mathrm{~b}$ ).

(a)

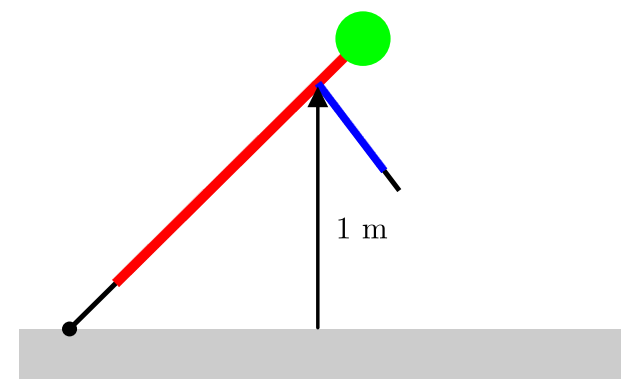

(b)

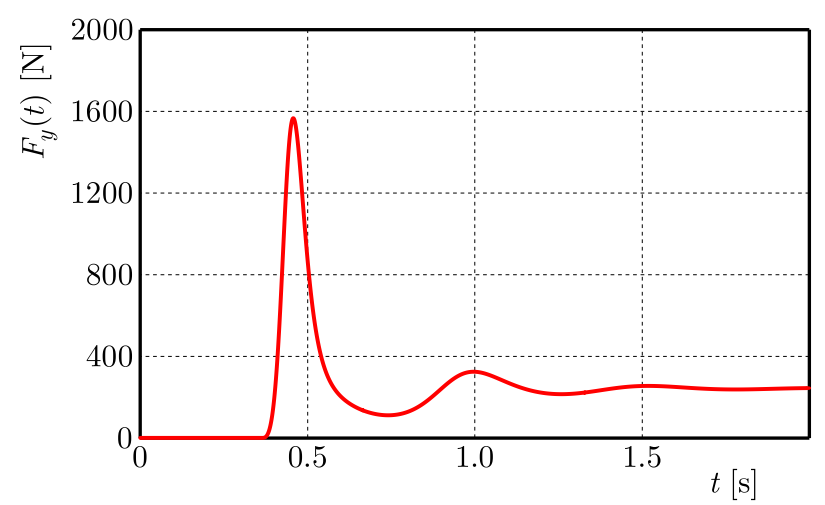

Fig. 5. Initial configuration of the faller's body, i.e. the fall from the shoulder height of $1 \mathrm{~m}$ (a), and the time history of GRF obtained numerically for the proposed forward fall model for $k_{y}=50000 \mathrm{~N} / \mathrm{m}^{3}$ and $b_{y}=0.6 \mathrm{~s} / \mathrm{m}$, which corresponds to the GRF obtained experimentally for a representative fall from the shoulder height of $1 \mathrm{~m}$ (b) (DeGoede and Ashton-Miller, 2003)

\section{Numerical simulation of the forward fall model}

\subsection{Parameters and relationships used in simulation}

Experimental observations confirmed that with an increase in the walking speed $v_{0}$, the human instinctively faster pulls his arms in the forward direction and the value of the mentioned parameter $\lambda$ also increases. This is why in all our numerical simulations we use the time histories 
of the angle $\theta_{2}(t)$ governed by Eq. (4.1) with different parameters $\theta_{2 \max }$ and $\lambda$, depending on the angle $\phi_{\text {Arm }}$ (the angle between the arm and the vertical axis of the Cartesian coordinate system at the moment of the impact to the ground) and the speed $v_{0}$ of the human walking just before the trip. Table 1 presents the dependence of the angle $\theta_{2 \max }$ on the angle $\phi_{A r m}$, while Table 2 presents the dependence of parameters $\lambda$ and $T$ on the speed $v_{0}$ of the faller before the trip. The parameters of the proposed fall model (identified in Section 4) allow one to conduct broader analysis for different parameters and different fall scenarios.

Table 1. Values of the angle $\theta_{2 \max }$ corresponding to different values of the angle $\phi_{\text {Arm }}$

\begin{tabular}{|c|c|c|c|c|c|c|c|}
\hline$\phi_{\text {Arm }}\left[^{\circ}\right]$ & 0 & 5 & 10 & 15 & 20 & 25 & 30 \\
\hline$\theta_{2 \max }\left[{ }^{\circ}\right]$ & 67.75 & 72.85 & 78.11 & 83.55 & 89.16 & 94.93 & 100.86 \\
\hline
\end{tabular}

Table 2. Values of parameters $T$ and $\lambda$ corresponding to different values of walking speed $v_{0}$

\begin{tabular}{|l|c|c|c|c|c|c|}
\hline$v_{0}[\mathrm{~m} / \mathrm{s}]$ & 0.5 & 1.0 & 1.5 & 2.0 & 2.5 & 3.0 \\
\hline$T[\mathrm{~s}]$ & 1.0 & 0.7 & 0.58 & 0.5 & 0.4 & 0.35 \\
\hline$\lambda[1 / \mathrm{s}]$ & 1.58 & 2.23 & 2.70 & 3.15 & 3.92 & 4.50 \\
\hline
\end{tabular}

\subsection{Time histories of ground reaction forces}

Figure 6 shows the time history of GRF obtained for a fall from the full standing position $\left(\theta_{1}(0)=90^{\circ}\right), v_{0}=1.5 \mathrm{~m} / \mathrm{s}$ and $\phi_{\text {Arm }}=15^{\circ}$, which corresponds to the most common falls met in real situations. In this case, the maximum value of GRF reaches $2400 \mathrm{~N}$, which corresponds to the distal radius fracture threshold (Kim and Ashton-Miller, 2009). Thus, we demonstrated that the taken typical and the most common falling conditions are enough to cause compressive fracture of the distal radius. The investigated falling process has been also observed by using the animation created in Mathematica (see Fig. 7).

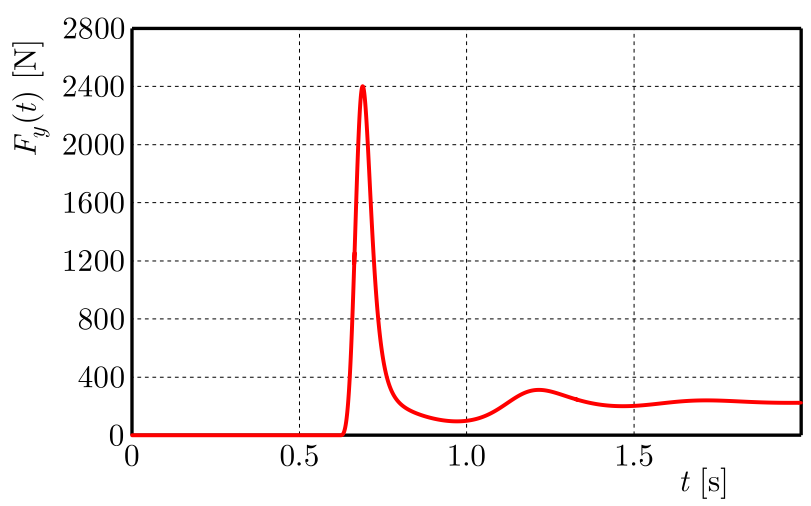

Fig. 6. Time histories of GRF from the standing position and $v_{0}=1.5 \mathrm{~m} / \mathrm{s}$
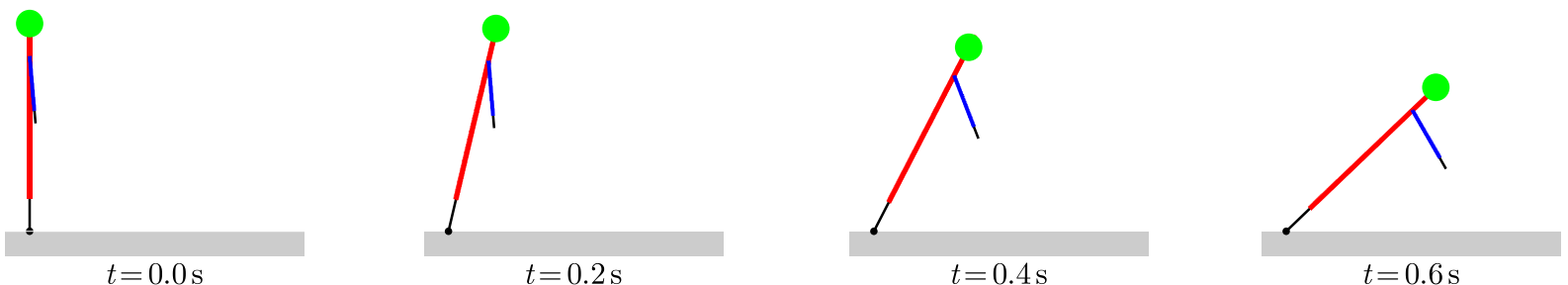

Fig. 7. Animation snapshots of the faller's body plotted in regular time intervals in different phases of the fall from the full standing position and walking speed $v_{0}=1.5 \mathrm{~m} / \mathrm{s}$ (obtained in Mathematica software) 
Figure 8 illustrates times histories of the force $F_{y}(t)$ acting on a single hand for $v_{0}=1.5 \mathrm{~m} / \mathrm{s}$ and for different values of the angle $\phi_{A r m}$. For all three presented cases, the plotted time histories of GRF are similar. However, it can be noticed that the maximum value of GRF increased from $2374 \mathrm{~N}$ for $\phi_{\text {Arm }}=0$ to $2486 \mathrm{~N}$ for $\phi_{\text {Arm }}=30^{\circ}$.

(a)

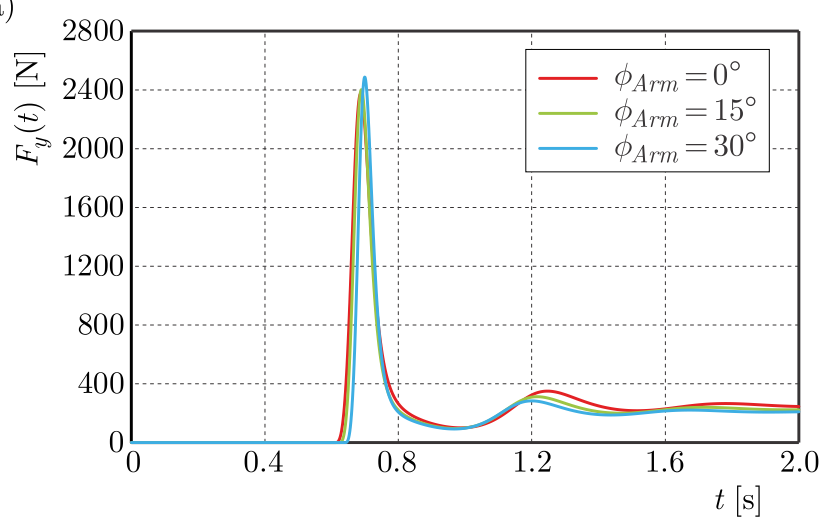

(b)

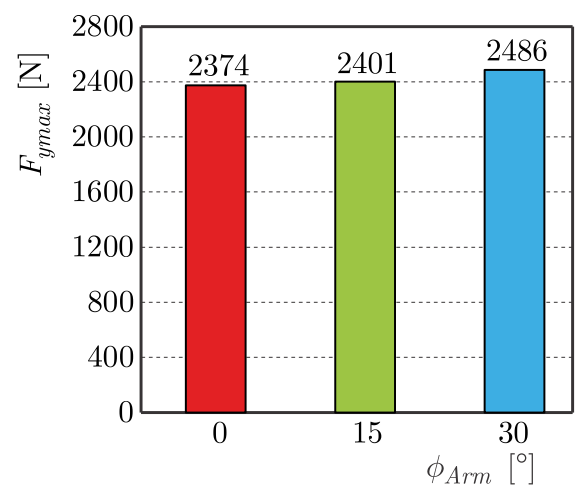

Fig. 8. GRFs for $v_{0}=1.5 \mathrm{~m} / \mathrm{s}$ and different values of angle $\phi_{A r m}$ : (a) time histories $F_{y}(t)$, (b) maximum values $F_{y} \max$

The results in Fig. 9 show the influence of different values of the velocity $v_{0}$ on time histories of the force $F_{y}(t)$. For larger values of the parameter $v_{0}$, the duration of the fall is smaller while the maximum value of GRF is greater. The value of GRF increases from $2246 \mathrm{~N}$ for $v_{0}=0.5 \mathrm{~m} / \mathrm{s}$ to $2534 \mathrm{~N}$ for $v_{0}=2.0 \mathrm{~m} / \mathrm{s}$. It means that for a smaller speed $v_{0}$ (i.e. $v_{0}<1.5 \mathrm{~m} / \mathrm{s}$ ), the GRF is less than the distal radius fracture threshold, whereas for larger values of $v_{0}$ (i.e. $v_{0}>1.5 \mathrm{~m} / \mathrm{s}$ ), this threshold is exceeded.

(a)

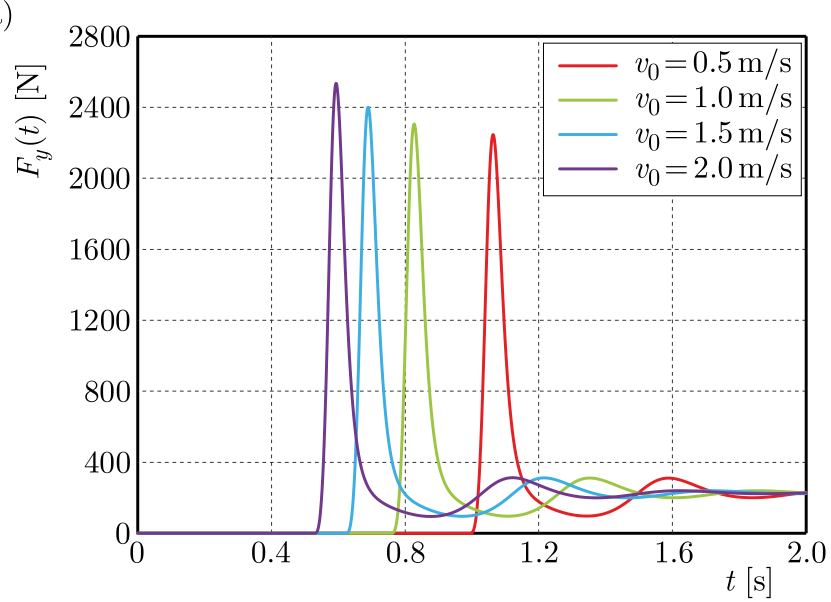

(b)

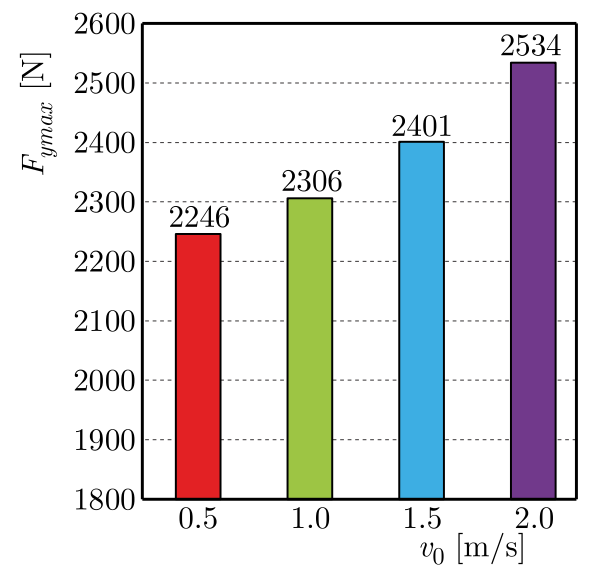

Fig. 9. GRFs for $\phi_{A r m}=15^{\circ}$ and different values of the velocity $v_{0}$ : (a) time histories $F_{y}(t)$, (b) maximum values $F_{y} \max$

Figure 10 presents maximum values of GRF as a function of the velocity $v_{0}$ for different values of the angle $\phi_{\text {Arm }}$. The maximum value of GRF increases with the increasing speed $v_{0}$ as well as the angle $\phi_{A r m}$. For the lowest presented value of $v_{0}$ equal to $0.5 \mathrm{~m} / \mathrm{s}$, the maximum value of GRF increases from $2217 \mathrm{~N}$ for $\phi_{A r m}=0$ to $2335 \mathrm{~N}$ for $\phi_{A r m}=30^{\circ}$. For the largest value of $v_{0}$ equal to $3.0 \mathrm{~m} / \mathrm{s}$, the maximum value of GRF increases from $2898 \mathrm{~N}$ for $\phi_{\text {Arm }}=0$ to $3000 \mathrm{~N}$ for $\phi_{\text {Arm }}=30^{\circ}$. If one considers the case $\phi_{\text {Arm }}=0$, the maximum value of GRF increases from $2217 \mathrm{~N}$ for $v_{0}=0.5 \mathrm{~m} / \mathrm{s}$ to $2898 \mathrm{~N}$ for $v_{0}=3.0 \mathrm{~m} / \mathrm{s}$. In the case of $\phi_{\text {Arm }}=30^{\circ}$, the maximum value of GRF increases from $2335 \mathrm{~N}$ for $v_{0}=0.5 \mathrm{~m} / \mathrm{s}$ to $3000 \mathrm{~N}$ for $v_{0}=3.0 \mathrm{~m} / \mathrm{s}$. 
For small values of $v_{0}\left(v_{0} \leqslant 1 \mathrm{~m} / \mathrm{s}\right)$, the maximum value of GRF is less than the distal radius fracture threshold, regardless of the angle $\phi_{A r m}$. In the case of $v_{0}=1.5 \mathrm{~m} / \mathrm{s}$, the maximum value of GRF is less than the mentioned fracture threshold when $0 \leqslant \phi_{\text {Arm }} \leqslant 10^{\circ}$, whereas GRF exceeds this threshold for $\phi_{A r m}>15^{\circ}$. For $v_{0} \geqslant 2.0 \mathrm{~m} / \mathrm{s}$, the maximum of GRF exceeds the distal radius fracture threshold regardless of the angle $\phi_{A r m}$. Concluding, for a small walking speed before the falling process, the value of the angle $\phi_{A r m}$ does not have a great influence. At a higher speed of human gait, the distal radius fracture threshold is usually exceeded, finally leading to injuries and/or fractures of the upper extremities. In the case of $v_{0}=1.5 \mathrm{~m} / \mathrm{s}$, the correct configuration of the human body (the correct value of the angle $\phi_{A r m}$ ) at the moment of the impact to the ground can reduce the maximum value of GRF and, as a result, can protect the faller from potential injuries or fractures of the upper extremity bones.

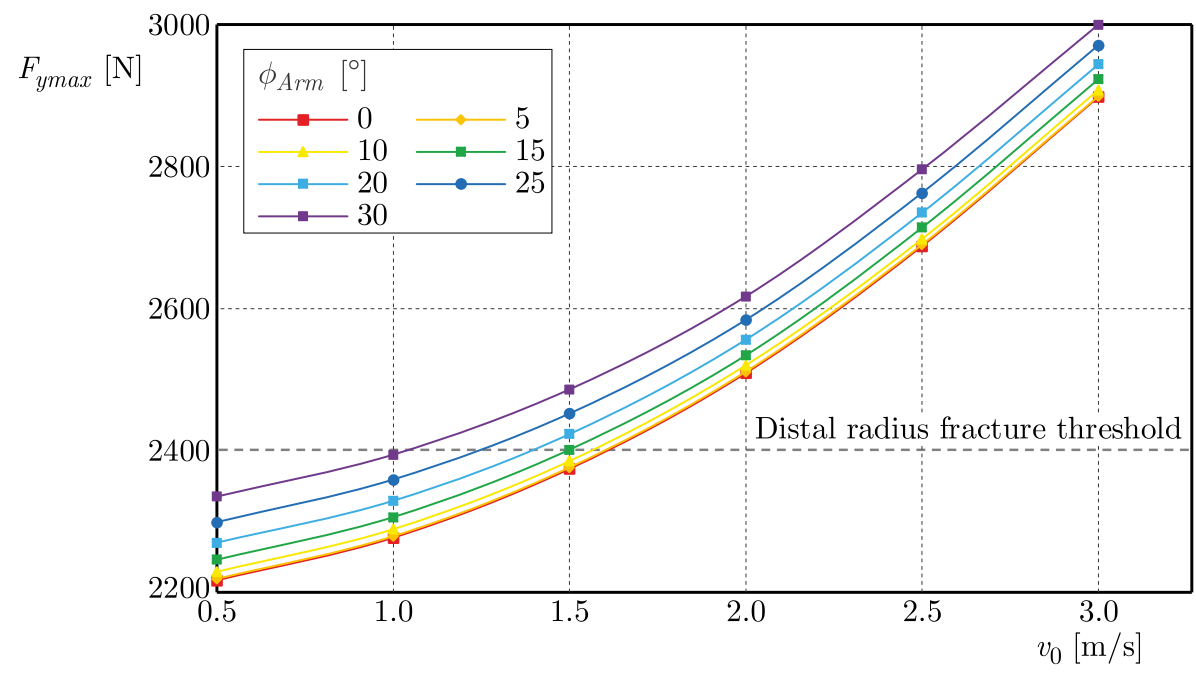

Fig. 10. Maximum values $F_{y} \max$ of GRF as a function of the velocity $v_{0}$ for different values of the angle $\phi_{\text {Arm }}$

\section{Finite element analysis of the radial bone}

\subsection{Geometry and material properties of the radial bone model}

The DICOM data used in this paper come from cadavers of 35-years-old man with a height of $1.73 \mathrm{~m}$ and weight of $75 \mathrm{~kg}$. These data have been obtained using a Siemens 64 Slice computed tomography (CT) Scanner in the Department of Forensic Medicine, Jagiellonian University Medical College, Cracow, Poland. The DICOM file of all upper extremity bones composed of the total number of 274 slices with the slice thickness equal to $1.5 \mathrm{~mm}$, pixel size equal to $0.977 \mathrm{~mm}$ and resolution $512 \times 512$ was imported to Mimics. During the next steps, the radius was separated, the computer model of this bone was obtained, and the computational mesh of the radius was corrected to avoid further numerical errors. As a result, the mesh of the radius was reduced to 3444 surface elements, and the correctness of the constructed computational mesh was verified using a Fix Wizard function. Finally, a realistic 3D FE model of the radius consisting of 15751 FEs was obtained and used for the strength analysis in Ansys. We used the SOLID185 FE-shaped tetrahedron element. The assumed in this paper isotropy of the material is not ideal, but the CT data provided only scalar information, and the determination of principal material directions had to be inferred (Neuert et al., 2013).

Material inhomogeneity of the radius was modelled in Mimics based on the CT images. First, we tested three different density-elasticity relationships describing human long bones to obtain the correct range of Young's modulus of the radius based on the CT images and the Hounsfield 
Unit scale. Finally, mechanical properties of the considered radial bone were calculated based on the density-elasticity relationships proposed by Rho et al. (1995)

$$
\rho=1.067 \mathrm{HU}+131 \quad E=0.004 \rho^{2.01}
$$

where $\rho$ expressed in $\left[\mathrm{kg} / \mathrm{m}^{3}\right]$ represents density of the bone, HU is the nondimensional Hounsfield Unit, $E$ expressed in [MPa] denotes Young's modulus, whereas $\nu$ is Poisson's ratio. The obtained range of Young's modulus of the radial bone is close to the range of the appropriate values for this bone presented in the literature. Young's moduli of the radius vary in the range 600-12000 MPa (see Fig. 11), whereas Poisson's ratio equals 0.3 for all FEs.
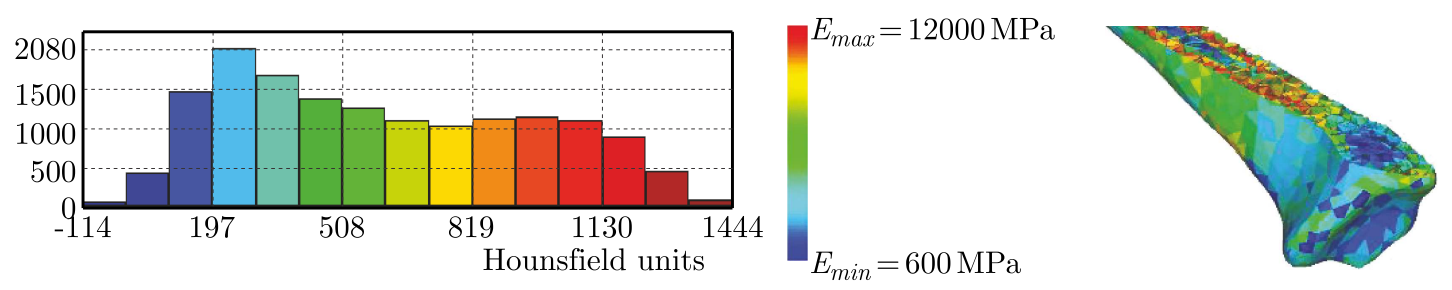

Fig. 11. Histogram and cross-sections of the radius showing quantitive distributions of FEs characterised by different Hounsfield Units and spatial distribution of inhomogeneous material properties

\subsection{Load and boundary conditions}

Numerical results presented in Fig. 6 were applied as the load conditions of the developed FE upper extremity model analysed in Ansys. These time histories of GRFs correspond to the GRF for the standing position and the walking speed $v_{0}=1.5 \mathrm{~m} / \mathrm{s}$. As far as the boundary conditions applied in the analysed numerical FE model of the radius were concerned, all six spatial DoFs in the proximal radius (region of the elbow joint) were fixed. The angle between the longitudinal axis of the radius and axis of the gravity field was equal to $\phi_{\text {Arm }}$, while the GRF was applied in the region of the radial neck in the vertical direction (direction of the gravity field). In all the investigated cases, we considered the same time-varying load condition (see Fig. 6), but the analysis was conducted for different directions of the GRF acting on the radius (i.e. for different angles $\phi_{\text {Arm }}$ ).

\subsection{Stress and strain analyses}

To predict bone fracture sites, we decided to measure the maximum von Mises stresses as the main criterion first. Moreover, we also measured maximal strains of the bones as another criterion leading to the additional information useful for a better understanding of the upper extremity behaviour under the applied load conditions, i.e. the assessment of the radius fracture site and failure load. The determination of the moment of time when the maximum stresses and strains were the highest enabled finding the fracture site and to investigate the state of the rest of the bones. The mentioned results are presented in Figs. 12 and 13 in the form of von Mises stress and strain distributions.

Values of both the maximal von Mises stress and bone strains significantly depend on the value of the angle $\phi_{A r m}$. Namely, with an- increase in the angle $\phi_{A r m}$, von Mises stresses and strains also increase. It should be noted that in the case of axial compression of the bone $\left(\phi_{A r m}=0\right)$, there are no bending torques and the bone itself is more resistant in such a configuration in comparison to the nonaxial compression. In the case of nonaxial GRF, bending torques are significant and, therefore, there are considerable values of both stresses and strains. For all the considered cases, the maximum values of stress and strain occur for $\phi_{\text {Arm }}=30^{\circ}$. 

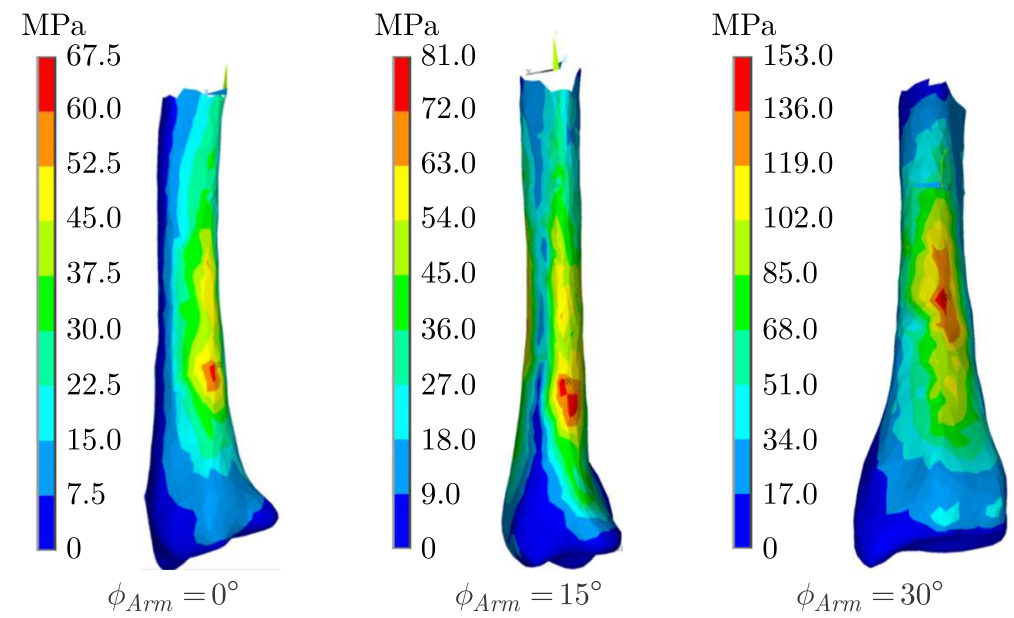

Fig. 12. Stress distributions in the radius for different angles $\phi_{\text {Arm }}$
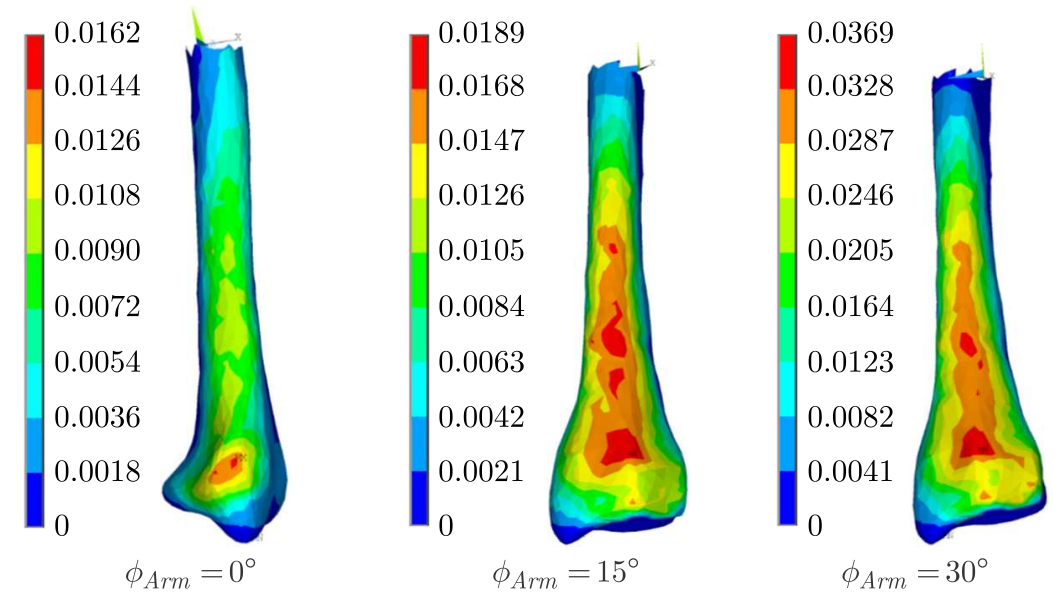

Fig. 13. Strain distributions in the radius for different angles $\phi_{A r m}$

The results presented in Figs. 12 and 13 show also other regularity. Namely, the maximum stresses occur on the medial side (diaphysis) of the radius bone while the maximum strains occur in the distal region of this bone. As it is known, Colles' fracture is the most common type of injury related to the forward fall on the outstretched upper extremities. Therefore, the presented results indicate that the strain criterion can be more useful for estimating the radius fracture site (the maximum strains are concentrated in the distal radius, see Fig. 13).

Table 3. Maximum von Mises stress and maximum strains for three different angles $\phi_{\text {Arm }}$

\begin{tabular}{|c|c|c|c|}
\cline { 3 - 4 } \multicolumn{2}{c|}{} & Radius & Time [s] \\
\hline \hline \multirow{2}{*}{$\phi_{\text {Arm }}=0$} & von Mises stress [MPa] & 65.54 & 0.122 \\
\cline { 2 - 4 } & Strain [-] & 0.0154 & 0.126 \\
\hline \multirow{2}{*}{$\phi_{\text {Arm }}=15^{\circ}$} & von Mises stress [MPa] & 78.88 & 0.132 \\
\cline { 2 - 4 } & Strain [-] & 0.0186 & 0.132 \\
\hline \multirow{2}{*}{$\phi_{\text {Arm }}=30^{\circ}$} & von Mises stress [MPa] & 148.58 & 0.124 \\
\cline { 2 - 4 } & Strain [-] & 0.0363 & 0.124 \\
\hline
\end{tabular}

In contrast to previous numerous papers dealing usually with resting conditions in our studies we carried out also a transient state analysis in Ansys. Values of maximum stresses and strains of the radius bone for different angles $\phi_{A r m}$ are presented in Table 3 . The maximum values of 
the von Mises stress and strains do not occur at the moment of the maximum peak of GRF but later, i.e. at the time about $0.12-0.13 \mathrm{~s}$.

\section{Conclusions}

The forward fall model proposed in this paper enables one to estimate the vertical ground reaction forces acting on the hands in various scenarios of the human falling process. The obtained numerical simulations fit with other results presented in the literature (Kim and Ashton-Miller, 2009), both from a qualitative and quantitative point of view. Moreover, the simulations show that the parameters describing the human body and parameters modelling biomechanical properties between the palmar cartilages and the ground have an essential influence on the obtained results.

It should be emphasized that the developed model has some limitations. First of all, the movement of the shoulder grid with respect of the torso and stiffness/damping properties of the shoulder joint have not been implemented in our model. Moreover, the horizontal component of the ground reaction force has not been considered. Nevertheless, the mentioned limitations may be of interest for our future study. On the other hand, our further modifications and improvements of the proposed model can be oriented on the increase of the number of DoFs of the mechanical model describing the human body as well as taking into consideration the rotary stiffness and damping properties in each of the human joints.

The choice of a linear material law is justified by small displacements of the radius bone observed during compressive tests presented in the literature (Bosisio et al., 2007). Although all bones have been modelled as a linear elastic isotropic material, in our opinion, the applied simplification should not significantly affect the ability of the proposed model to predict fracture sites and failure load of the radius bone under loads resulting from a forward fall. The variability of Poisson's ratio has not been evaluated in our investigations.

The performed numerical investigations with the time history of the GRF that occur during the falling process in the forward direction on the outstretched arms are sufficient to determine potential fracture sites and the obtained results agree with numerical/experimental results presented in the literature (Edwards and Troy, 2012). In addition, the obtained results indicate that the angle $\phi_{\text {Arm }}$ and, consequently, the direction of load applied to the radius have a strong impact on the fracture strength of this bone. It means that falls from a standing position on the outstretched arms generate the value of GRF which can exceed the mean human distal radius fracture threshold. Moreover, we have also shown that the maximal strain criterion seems to be more useful for the estimation of the fracture site than the appropriate von Mises stress criterion. Although we obtained various numerical results, unfortunately we were unable to compare these results with own experimental studies from a quantitative point of view. However, the obtained numerical results show that our model provides a realistic estimation of radius bone strain, fracture strength and fracture side estimation under various loading scenarios simulating a forward fall.

\section{Ethical Approval}

This article does not contain any studies performed on animals. The presented experimental studies have been performed using one of the author of this paper (Paweł Biesiacki) without any other human participants.

\section{Acknowledgments}

The work has been partially supported by the National Science Centre of Poland under the grant OPUS 9 No. 2015/17/B/ST8/01700 for years 2016-2018. 


\section{References}

1. Bosisio M.R, Talmant M., Skalli W., Laugier P., Mitton D., 2007, Apparent Young's modulus of human radius using inverse finite-element method, Journal of Biomechanics, 40, $2022-2028$

2. Burkhart T.A., Andrews D.M., Dunning C.E., 2013, Multivariate injury risk criteria and injury probability scores for fractures to the distal radius, Journal of Biomechanics, 46, 973-978

3. Chiu J., Robinovitch S.N., 1998, Prediction of upper extremity impact forces during falls on the outstretched hand, Journal of Biomechanics, 31, 1169-1176

4. Davidson P.L., Chalmers D.J., Stephenson S.C., 2006, Prediction of distal radius fracture in children, using biomechanical impact model and case-control data on playground free falls, Journal of Biomechanics, 39, 503-509

5. DeGoede K.M., Ashton-Miller J.A., 2002, Fall arrest strategy affects peak hand impact force in a forward fall, Journal of Biomechanics, 35, 843-848

6. DeGoede K.M., Ashton-Miller J.A., 2003, Biomechanical simulations of forward fall arrests: effects of upper extremity arrest strategy, gender and aging-related declines in muscle strength, Journal of Biomechanics, 36, 413-420

7. Edwards W.B., Troy K.L., 2012, Finite element prediction of surface strain and fracture strength at the distal radius, Medical Engineering and Physics, 34, 290-298

8. FrYkMAn G., 1967, Fracture of the distal radius including sequelae shoulder-hand-finger syndrome, disturbance in the distal radio-ulnar joint and impairment of nerve function, Acta Orthopaedica Scandinavica, 108, 1-153

9. Gerritsen K.G.M., van Den Bogert A.J., Nigg B.M., 1995, Direct dynamics simulation of the impact phase in heel-toe running, Journal of Biomechanics, 28, 661-668

10. GrabCAD, 2016, Open CAD library (https://grabcad.com/library)

11. Heijnen M.J.H., Rietdyk S., 2016, Falls in young adults: Perceived causes and environmental factors assessed with a daily online survey, Human Movement Science, 46, 86-95

12. Johnell O., Kannis J.A., 2006, An estimate of the worldwide prevalence and disability associated with osteoporotic fractures, Osteoporosis International, 17, 1726-1733

13. Kim K.-J., Ashton-Miller J.A., 2009, Segmental dynamics of forward fall arrests: A system identification approach, Clinical Biomechanics, 24, 348-354

14. Kroemer K.H.E., Kroemer H.J., Kroemer-Elbert K.E., 1997, Engineering Physiology: Bases of Human Factors/Ergonomics, Van Nostrand Reinhold, New York

15. Neuert M.A.C., Austman R.L., Dunning C.E., 2013, The comparison of density-elastic modulus equations for the distal ulna at multiple forearm positions: a finite element study, Acta of Bioengineering and Biomechanics, 15, 37-43

16. Nevitt M.C., Cummings S.R., 1993, Type of fall and risk of hip and wrist fractures: the study of osteoporotic fractures, Journal of the American Geriatrics Society, 41, 11, 1226-1234

17. O’Neill T.W., Varlow J., Silman A.J., Reeve J., Reid D.M., Todd C., Woolf A.D., 1994, Age and sex influences on fall characteristics, Annals of the Rheumatic Diseases, 53, 773-775

18. Palvanen M., Kannus P., Parkkari J., Pitkajarvi T., Pasanen M., Vuori I., Jarvinen M., 2000, The injury mechanisms of osteoporotic upper extremity fractures among older adults: a controlled study of 287 consecutive patients and their 108 controls, Osteoporosis International, 11, $822-831$

19. Rно J.Y., Новатно M.C., Ashman R.B., 1995, Relations of mechanical properties to density and CT number in human bone, Medical Engineering and Physics, 17, 347-355 
20. Robinovitch S.N., Feldman F., Yang Y., Schonnop R., Leung P.M., Sarraf T., SimsGould J., Loughin M., 2013, Video capture of the circumstances of falls in elderly people residing in long-term care: an observational study, The Lancet, 381, 47-54

21. Spadaro J.A., Werner F.W., Brenner R.A., Fortino M.D., Fay L.A., Edwards W.T., 1994, Cortical and trabecular bone contribute strength to the osteopenic distal radius, Journal of Orthopaedic Research, 12, 211-218

22. Vellas B.J., Wayne S.J., Garry P.J., Baumgartner R.N., 1998, A two-year longitudinal study of falls in 482 community-dwelling elderly adults, The Journals of Gerontology, Series A, Biological Sciences and Medical Sciences, 53, 264-274

Manuscript received June 7, 2017; accepted for print September 21, 2017 